\title{
Bauen mit
}

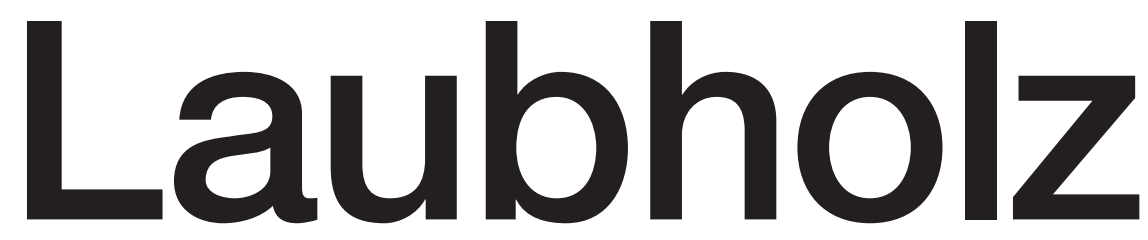

Konrad Merz

Anne Niemann

Stefan Torno 


\section{Autoren}

Konrad Merz

Anne Niemann

Stefan Torno

mit Beiträgen von:

Hermann Kaufmann (Vorwort)

Markus Lechner (Hochleistungswerkstoffe mit Potenzial für die Zukunft)

Stefan Winter (Hochleistungswerkstoffe mit Potenzial für die Zukunft)

\section{Verlag}

Redaktion, Layout und Lektorat:

Steffi Lenzen (Projektleitung und Texte Projektbeispiele);

Claudia Fuchs (Projektbeispiele), Jana Rackwitz (Theoriekapitel)

Redaktionelle Mitarbeit:

Charlotte Petereit

Korrektorat:

Sandra Leitte

Coverdesign nach einem Konzept von: Kai Meyer

Zeichnungen:

Rana Aminian, Ralph Donhauser, Sandra Gunnermann, Martin Hämmel, Ursula Sparakowski

Herstellung und Produktion:

Simone Soesters

Reproduktion:

ludwig:media, Zell am See (AT)

Druck und Bindung:

Grafisches Centrum Cuno GmbH \& Co. KG, Calbe (DE)

Papier:

Peydur lissé (Umschlag), Profibulk (Innenteil)

Verlag:

DETAIL Business Information GmbH

Messerschmittstr. 4, 80992 München

detail.de

๑ 2020, erste Auflage

ISBN 978-3-95553-504-9 (Print)

ISBN 978-3-95553-505-6 (E-Book)

Bibliografische Information der Deutschen Nationalbibliothek

Die Deutsche Nationalbibliothek verzeichnet diese Publikation in der Deutschen Nationalbibliografie; detaillierte bibliografische Daten sind im Internet abrufbar über http://dnb.d-nb.de.

Dieses Werk ist urheberrechtlich geschützt. Die dadurch begründeten Rechte, insbesondere die der Übersetzung, des Nachdrucks, des Vortrags, der Entnahme von Abbildungen und Tabellen, der Funksendung, der Mikroverfilmung oder der Vervielfältigung auf anderen Wegen und der Speicherung in Datenverarbeitungsanlagen, bleiben, auch bei nur auszugsweiser Verwertung, vorbehalten. Eine Vervielfältigung dieses Werks oder von Teilen dieses Werks ist auch im Einzelfall nur in den Grenzen der gesetzlichen Bestimmungen des Urheberrechtsgesetzes in der jeweils geltenden Fassung zulässig. Sie ist grundsätzlich vergütungspflichtig. Zuwiderhandlungen unterliegen den Strafbestimmungen des Urheberrechts.

Dieses Fachbuch berücksichtigt die bei Redaktionsschluss gültigen Begriffe und den zu diesem Zeitpunkt aktuellen Stand der Technik. Rechtliche Ansprüche können aus dem Inhalt dieses Buchs nicht abgeleitet werden. Für Vollständigkeit und Richtigkeit aller Beiträge wird keine Gewähr übernommen. 